\title{
Addition of $\alpha, \alpha$-Difluoroiodomethyl Ketones to Alkenes with a Copper Catalyst
}

\author{
Kyung Chell Kwak, Woo-Yiel Lee, Quan Zheshan, ${ }^{\star}$ Young Hang Lee, Young-Gab Yun, ${ }^{\ddagger}$ \\ Gyu Beum Kwak, ${ }^{,}$Hun-Taeg Chung," Tae-Oh Kwon," and Kyu Yun Chai"
}

\author{
Department of Bionamochemistry and Basic Sciences Research Insitute, Wonkwang University, Iksan, Chonbuk 570-749, Korea \\ ${ }^{+}$E-mail: genvon'à wonkwang. ac: kr \\ "College of Phamacy: Yanbian Lniversity; Yami 133000, China \\ "Department of Prescription, School of Oriental Medicine, Wonkwang University, Iksan. Chonbuk 570-749, Korea \\ ${ }^{\$}$ Department of New Material Science, Chonbuk Natonal Unversity, Jeoniu. Chonbuk 561-756. Korea \\ Medicinal Resources Research Center of Wonkwang Liversity, Iksan, Chonbuk 570-749. Korea \\ 'College of Life Science and Natural Resources, Wonkwang Unversity, Iksan, Chonbuk 570-749. Korea
}

Received.Jily 20,2004

\begin{abstract}
The addition reactions of $\alpha$. $\alpha$-difluoroiodomethyl n-butyl ketone, $\alpha, \alpha$-difluoroiodomethyl cyclohexyl ketone, or $\alpha, \alpha$-difluoroiodomethyl phenyl ketone to alkenes were successfully accomplished in good yields in the presence of copper powder. The reaction was also applicable to alkenes containing a variety of functional groups such as ester, trimethylsilyl, or ether group. Acetonitrile was determined to be the best solvent in the present study and the reaction was performed at $55^{\circ} \mathrm{C}$ for $15-22 \mathrm{~h}$. This reaction provides a new, efficient and general method for the synthesis of $\alpha, \alpha$-difluoro functionalized ketones.
\end{abstract}

Key Words : $\alpha, \alpha$-Difluoroiodomethyl ketone derivatives, Copper catalyst

\section{Introduction}

The orgatic compounds with selectively introduced difluoromethylene group have been known to exhibit important biological properties such as the inhibitory effects on various enzymes. ${ }^{1}$ antibiotic, ${ }^{2}$ anti-human immunodeficiency virus (HIV), ${ }^{3}$ anticancer and antihypertensive effects. ${ }^{\prime}$

Difluoromethylene group has been generally introdued to organics by the methods previously reported as follows. The addition of perfluoroalkyl iodide to alkenes was performed in the presence of various catalysts such as titanium, ${ }^{5}$ benzoylperoxide, ${ }^{6}$ sodium dithionite ${ }^{7}$ and organophosphines. ${ }^{8}$ The Reformatsky reaction was used to prepare $\alpha$, $\alpha$-difluoro$\beta$-hydroxy carbonyl compounds from the reaction of aldehydes with $\alpha$-chloro- $\alpha$. $\alpha$-difluoromethyl ketones" or $\alpha$ bromo- $\alpha, \alpha$-difluoro acetates. ${ }^{10}$ The addition of ethyl bromodifluoroacetate to alkenes was carried out using nikel chloride or copper powder as a catalyst. "The transfornation of carbonyl group to difluoromethylene group utilizing (diethylamino)sulfur trifluoride (DASl) was reported. ${ }^{12}$ In addition, difluoromethylene ketone was recently reported to be introdued to the electron-deficient olefins using UV irradiation, 1.3 while palladium catalyst was used for the addition to electron-rich olefins. ${ }^{\text {l.t }}$

However, since the experimental procedure recuired for the photoreaction involving the repeated freezing under liquid nitrogen and degassing processes is complicated, this method could be limited in general utilization. Moreover, palladium is expensive and limited numbers of method are available for the addition of difluoromethylene group to electron-rich olefins. As a preliminary result, we briefly described the addition of $\alpha, \alpha$-difluoroiodomethyl cyclohexyl ketone to alkenes in the presence of copper. ${ }^{15}$ We report here the detailed results concerning the addition of $\alpha, \alpha$-difluoroiodomethyl phenyl ketone, $\alpha, \alpha$-difluoroiodomethyl cyclohexy] ketone and $\alpha, \alpha$-difluoroiodomethyl $n$-butyl ketone to alkenes, respectively, with a variety of functional groups such as trimethylsilyl, ether and ester group in the presence of copper.

\section{Results and Discussion}

The starting compounds in this study, $\alpha . \alpha$-difluoroiodomethyl phenyl ketone (2a), $\alpha, \alpha$-difluoroiodomethyl cyclohexyl ketone (2b) and $\alpha, \alpha$-difluoroiodomethyl $n$-butyl ketone (2c), are readily obtained from chlorodifluoroacetic acid (1) using the Grignard reaction followed by the Reformatsky reaction ${ }^{16}$ (Scheme 1). The difluoroiodomethyl ketone compounds were reacted with alkenes having various functional groups (Scheme 2) such as vinyltrimethylsilane (3a), ethyl acrylate (3b), vinyl-1,3-dioxolane (3c), 3,3diethoxy-1-propene (3d), methyl 3-butenoate (3e), allyl phenyl ether ( $\mathbf{3 f}$ ) and allyl propyl ether $(\mathbf{3 g})$. The addition of 1.2 to 2 equivalents of olefins to $\alpha_{+} \alpha$-difluoroiodomethyl ketones (1a, Ib, Ic) was successfully carried out in acetonitrile at $55^{\circ} \mathrm{C}$ for 15 to $22 \mathrm{~h}$ in the presence of 30 mole

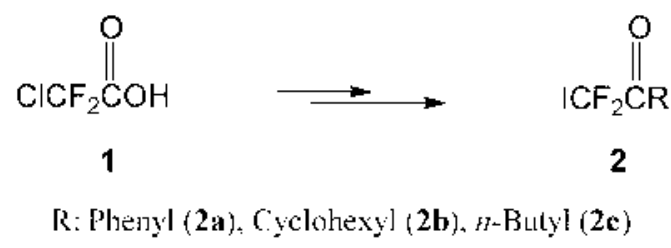

Scheme 1 


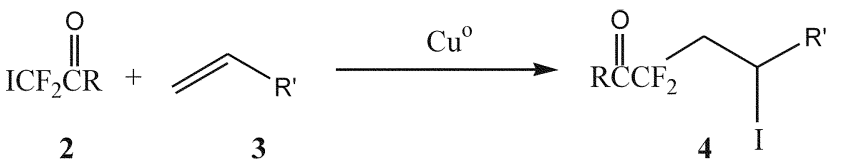

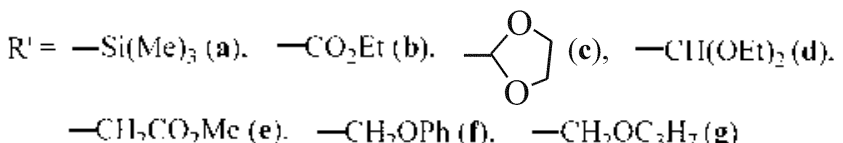

Scheme 2

Table 1. Copper-cataly red addition of difluoroindomethyl ketones to alkene derivatives

\begin{tabular}{|c|c|c|c|c|c|}
\hline Innlry & Substrate & $\begin{array}{l}\text { Reactant } \\
\text { (R') }\end{array}$ & $\begin{array}{l}\text { Reaction } \\
\text { time (h) }\end{array}$ & Product & $\begin{array}{l}\text { Yield } \\
(\%)\end{array}$ \\
\hline 1 & $1 \mathrm{a}$ & $3 a$ & 15 & 4aa & 97 \\
\hline 2 & 1a & $3 \mathbf{b}$ & 22 & $4 a b$ & 42 \\
\hline 3 & 1a & $3 c$ & 18 & $4 a c$ & 89 \\
\hline 4 & 1a & $3 d$ & 18 & 4ad & 73 \\
\hline 5 & $1 a$ & $3 e$ & 18 & 4ae & 78 \\
\hline 6 & 1a & $3 f$ & 15 & 4af & 95 \\
\hline 7 & $1 \mathrm{a}$ & $3 g$ & 18 & 4ag & 81 \\
\hline 8 & Ib & $3 a$ & 15 & $4 \mathrm{ba}$ & 90 \\
\hline 9 & Ib & $3 \mathrm{~h}$ & 22 & $4 \mathrm{bb}$ & 52 \\
\hline 10 & Ib & $3 c$ & 18 & $4 \mathrm{bc}$ & 80 \\
\hline 11 & Ib & $3 d$ & 18 & $4 \mathrm{bd}$ & 72 \\
\hline 12 & Ib & $3 e$ & 18 & 4 be & 82 \\
\hline 13 & Ib & $3 f$ & 22 & 4 bf & 66 \\
\hline 14 & Ib & $3 \mathrm{~g}$ & 22 & $4 \mathrm{hg}$ & 70 \\
\hline 15 & Ic & $3 \mathbf{a}$ & 15 & $4 \mathrm{ca}$ & 94 \\
\hline 16 & Ic & $3 \mathbf{b}$ & 22 & $4 \mathrm{cb}$ & 55 \\
\hline 17 & Ic & $3 c$ & 18 & $4 \mathrm{cc}$ & 85 \\
\hline 18 & Ic & $3 d$ & 18 & $4 \mathrm{~cd}$ & 74 \\
\hline 19 & Ic & $3 e$ & 18 & $4 \mathrm{ce}$ & 75 \\
\hline 20 & Ic & $3 f$ & 18 & $4 \mathrm{ef}$ & 88 \\
\hline 21 & Ic & $3 \mathrm{~g}$ & 18 & $4 \mathrm{cg}$ & 80 \\
\hline
\end{tabular}

$\%$ copper powder. The reaction products were obtained with 40 to $97 \%$ yields (Table 1 ).

All the products were identified using 'H NMR, ${ }^{15} \mathrm{C} N M R$, ${ }^{10} \mathrm{~F}$ NMR and MS spectra. ${ }^{19} \mathrm{~F}$ NMR spectra of all the adducts showed typical AB splitting patterns, because the two fluorines are not equivalent due to the presence of the $\gamma$-positioned chiral center. For example, the ${ }^{19} \mathrm{~F}$ NMR of 2,2-fluoro-4-iodo-l-phenyl-4-trimethylsilylbutanone had doublets at -109.2 and $-103.8 \mathrm{ppm}$ with coupling constants of ${ }^{2} J_{i \%}=261,0,{ }^{3} J_{i \%}=16.1$ and ${ }^{3} J_{i \%}=16.1 \mathrm{H}$.

The addition products formed from the reaction of $\alpha, \alpha$ difluoromethylphenyl ketone with various alkenes with the exception of ethyl acrylate were obtained with slightly higher yields (3-5\%) than those formed from $\alpha . \alpha$-difluoroiodomethyl cyclohexyl ketone or $\alpha, \alpha$-difluoromethyl $n$-butyl ketone. Considering the functional groups of alkene, the reaction with the electron-rich olefin (3a) having trimethylsilyl group gave the addition product in the highest yield $(>90 \%)$. By contrast, the electron-deficient olefin (3b) afforded the lower yields of the adducts in the reaction with the difluoroiodomethyl ketones (2). These results indicate that the mechanism of addition reaction has a similarity to that suggested for the reaction of iododifluoromethyl ketones with normal alkenes using $\mathrm{Pd}(0)$ catalyst. ${ }^{1+.17}$ Therefore, electron scavengers or radical inhibitors are expected to inhibit the addition reaction including a single electron transfer process. The radical mechanisin was supported by the observation that $\mathbf{2 a}$ was not reacted with $\mathbf{3 a}$ under the same reaction conditions in the presence of 30 mole $\%$ dinitrobenzene. The detail mechanism of the current reactions has been proposed in Figure 1 .

In order to improve the yield of addition, the reaction of $\alpha . \alpha$-difluoroiodomethyl phenyl ketone (2a) with trimethylsilane (3a) in presence of copper was allowed in a variety of solvents. When methylene chloride, acetonitrile, $n$-hexane, IHF, DMDO, DMF and ethyl acetate were employed for the reaction, acetonitrile was determined to be the best solvent. It is noteworthy that the coordinating solvents such as DMSO, DMF' and 'THF' gave low yields ( Cable 2).

Scince the fluorination of biologically important molecules has resulted in dramatic changes and distintive modifications in their biological activities, ${ }^{18}$ many efforts have been made to design the efficient methods for the synthesis of selectively fluorinated compounds. Although the recent methods using $\mathrm{P} d(0)$ catalyst and UV irradiation may be general ways to prepare the adducts of difluoroiodomethyl

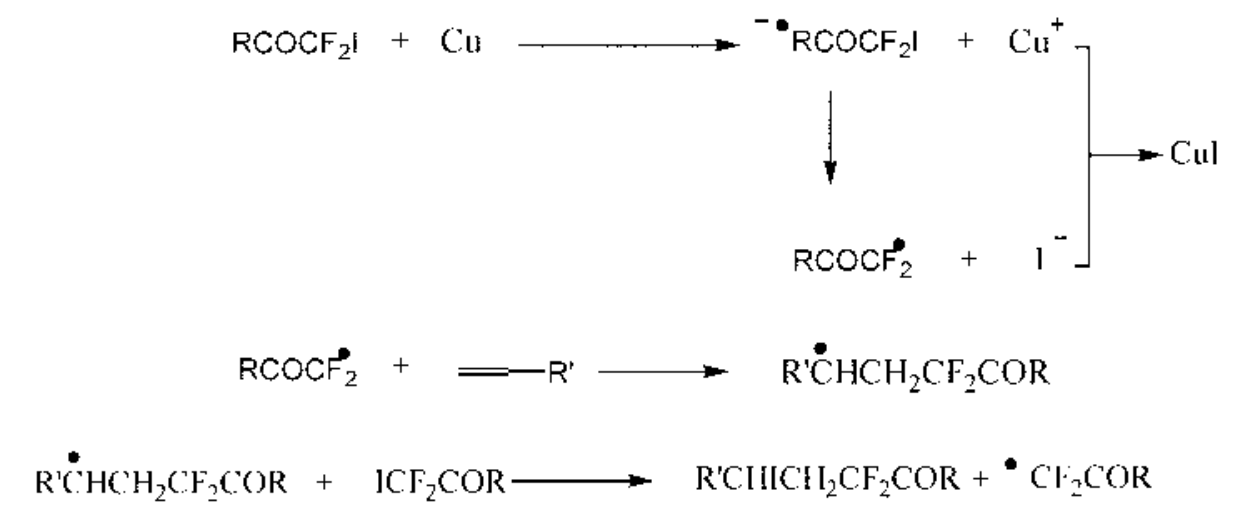

Figure 1. "I he proposed mechanism of the addition of $\alpha, \alpha$-difluoroiodomethyl alkyl ketones to alkenes in the presence of copper catalyst. 
Table 2. Investigation of solvent effect

\begin{tabular}{clcc}
\hline No & Solvent & Time $(\mathrm{h})$ & Yield $(\%)$ \\
\hline 1 & $\mathrm{CH}_{3} \mathrm{CN}$ & 18 & 99 \\
2 & $\mathrm{MC}$ & 18 & 60 \\
3 & $\mathrm{THF}$ & 18 & 30 \\
4 & $\mathrm{DMSO}$ & 6 & 40 \\
5 & $\mathrm{DMF}$ & 6 & 30 \\
6 & $\mathrm{EA}$ & 18 & $<5$ \\
7 & n-Hexane & 24 & $\mathrm{NR}$ \\
\hline
\end{tabular}

ketones with olefins. this method provides a simple and more efficient approach to the introduction of $\alpha . \alpha$ difluoromethylene group to various olefuns.

\section{Experimental Section}

General experimental procedures. NMR spectra were recorded in $\mathrm{CDCl}_{3}$ using a JEOL Eclipse $500-\mathrm{MHz}$ spectrometer, and ${ }^{1} \mathrm{H}$ and ${ }^{13} \mathrm{C}$ chemical shifts were referenced relative to the corresponding residual solvent signals ( $\delta 7.24 /$ 77.0). ${ }^{19} \mathrm{~F}$ NMR spectra were referenced relative to an internal $\mathrm{CFCl}_{3}$. All mass spectra analyses were performed at $70 \mathrm{eV}$ in electron impact mode. The IR spectra were obtained on a JASCO FT/IR-5300.

General procedure used to prepare the addition products (4). To a solution of $\alpha \alpha$-difluoroiodomethyl ketone (2. $0.1 \mathrm{~mol}$ ) and activated copper powder $(0.015 \mathrm{~mol})$ in dry acetonitrile $(100 \mathrm{~mL})$. olefun $(3,0.12$ to $0.20 \mathrm{~mol})$ was dropped under $\mathrm{N}$ : gas (Scheme 2). and the mixture was heated at $60^{\circ} \mathrm{C}$ for 15 to $22 \mathrm{~h}$. The reaction mixture was cooled to room temperature. and the solvent was evaporated. The resulting residue was partitioned between diethyl ether and water. and the diethyl ether layer was dried with $\mathrm{MgSO}_{4}$. After the solvent was evaporated under reduced pressure. the residue was applied onto a silica gel column to afford compound 4 .

2,2-Difluono-4-iodo-1-phenyl-4-trimethylsilyl-1-butanone (4aa). ${ }^{1} \mathrm{H} \mathrm{NMR}\left(\mathrm{CDCl}_{3}\right.$. TMS): $\delta 8.11$ (d. $J=7.79 \mathrm{~Hz} .2 \mathrm{H}$ ). $7.64(\mathrm{t} . J=7.33 \mathrm{~Hz}, 1 \mathrm{H}) .7 .50(\mathrm{t} . J=7.79 \mathrm{~Hz}, 2 \mathrm{H}) .3 .25(\mathrm{dd}$. $J=10.54,2.75 \mathrm{~Hz}, 1 \mathrm{H}) .2 .75(\mathrm{~m} .2 \mathrm{H}) .0 .20(\mathrm{~s} .9 \mathrm{H}){ }^{19} \mathrm{~F}$ NMR $\left(\mathrm{CDCl}_{3}, \mathrm{CFCl}_{3}\right)$ : $\delta-108.14$ (ddd, $J=264.37,17.04$. $17.0 \mathrm{~Hz} . \mathrm{IF}) \delta-104.78$ (ddd, $J=264.37,17.04 .17 .0 \mathrm{~Hz}$. 1F). $\left.{ }^{13} \mathrm{C} \mathrm{NMR} \mathrm{(CDCl}, \mathrm{TMS}\right): \delta 189.4$ (t. $J=30.7 \mathrm{~Hz}$ ). 134.6 (s). 132.2 (s), 130.5 (s), 128.9 (s). 119.3 (t, $J=256.2$ Hz). 38.3 (t. $J=23.0 \mathrm{~Hz}), 4.4(\mathrm{~s})$. GC-MS (m/e. relative intensity): $382\left(\mathrm{M}^{-}, 1.03\right) .105$ (48.34). 77 (96.60). 73 (100). IR $\left(\mathrm{CCl}_{4}\right): 3067.2955 .2926 .2854$. 1701. 1599. 1450. $125 \mathrm{l}$. $1417.1179 \mathrm{~cm}^{-1}$

Ethy1-4,4-difluoro-2-iodo-5-ox0-5-phenylpentanoate (4ab). ${ }^{1} \mathrm{H}$ NMR $\left(\mathrm{CDCl}_{3}\right) ; \delta 8.05$ (d. $\left.J=8.25 \mathrm{~Hz}, 2 \mathrm{H}\right), 7.62$ (t. $J=7.33 \mathrm{~Hz}, \mathrm{IH}) .7 .47$ (t, $J=7.79 \mathrm{~Hz} .2 \mathrm{H}$ ), 4.63 (dd. $J=10.77 .3 .44 \mathrm{~Hz} .1 \mathrm{H}) 4.20(\mathrm{q} . J=7.10 \mathrm{~Hz} .2 \mathrm{H}) 3.36(\mathrm{~m}$. 1H) $2.85(\mathrm{~m}, 1 \mathrm{H}), 1.17(\mathrm{t} . J=7.10 \mathrm{~Hz}, 3 \mathrm{H}) \cdot{ }^{1 \mathrm{~g}} \mathrm{~F} \mathrm{NMR}$ $\left(\mathrm{CDCl}_{3}, \mathrm{CFCl}_{3}\right): \delta-109.27$ (ddd. $J=261.48,17.01,17.0 \mathrm{l}$ $\mathrm{Hz}, 1 \mathrm{~F}$ ) $\delta-104.32$ (ddd, $J=261.48,17.01,17.01 \mathrm{~Hz}, \mathrm{IF}$ ). ${ }^{13} \mathrm{C} \mathrm{NMR}\left(\mathrm{CDCl}_{3}\right): \delta 187.93(\mathrm{t} . J=30.23 \mathrm{~Hz}) .134 .74(\mathrm{~s})$. $131.35(\mathrm{~s}) .130 .26(\mathrm{t} . J=3.84 \mathrm{~Hz}) 128.85(\mathrm{~s}), 118.35(\mathrm{t}$. $J=256.24 \mathrm{~Hz}) .62 .26(\mathrm{~s}), 41.21(\mathrm{t} . J=23.09 \mathrm{~Hz}) 13.69(\mathrm{~s})$, $7.99(\mathrm{t}, J=3.84 \mathrm{~Hz})$. IR $\left(\mathrm{CCl}_{4}\right): 3422.1740 .1449 \mathrm{~cm}^{-1}$. GC-MS ( $\mathrm{m} / \mathrm{e}$. relative intensity): $382\left(\mathrm{M}^{-} .0 .45\right) .337$ (24.26). 309 (27.24). $255(87.67) .182(29.36)$. $162(44.04)$, 133 (17.65). 105 (100).

2,2-Difluoro-4-(1,3-dioxolan-2-yl)-4-jodo-1-phenyl-1butanone (4ac). ${ }^{1} \mathrm{H}$ NMR $\left(\mathrm{CDCl}_{3}\right): \delta 8.10$ (d. $J=7.79 \mathrm{~Hz}$, $2 \mathrm{H}$ ). 7.64 (t, $J=7.33 \mathrm{~Hz}, 1 \mathrm{H}$ ). $7.5 \mathrm{I}$ (t, $J=7.79 \mathrm{~Hz}, 2 \mathrm{H}$ ), 4.68 (d. $J=3.21 \mathrm{~Hz} \mathrm{lH}), 4.38(\mathrm{ml} \mathrm{lH}), 4.11(\mathrm{~m} .2 \mathrm{H}), 3.97$ (m, 2H). 3.02. (dddd $J=18.83 \mathrm{~Hz}, 18.83 \mathrm{~Hz} .6 .88 \mathrm{~Hz}, 6.88$ $\mathrm{Hz} 2 \mathrm{H}$ ). ${ }^{19} \mathrm{~F} \mathrm{NMR}\left(\mathrm{CFCl}_{3}\right): \delta-107.34$ (ddd. $J=273.04$, 16.07, $16.07 \mathrm{~Hz}$. IF), $\delta-104.42$ (ddd. $J=273.04$. 16.07, $16.07 \mathrm{~Hz}, \mathrm{IF}) .{ }^{13} \mathrm{C}$ NMR $\left(\mathrm{CDCl}_{3}\right): \delta 188.53$ (t. $J=31.67$ $\mathrm{Hz}), 134.53(\mathrm{~s}) .131 .75(\mathrm{~s}), 130.27(\mathrm{t}, J=2.88 \mathrm{~Hz}), 128.80$ (s), 118.67 (t. $J=256.24 \mathrm{~Hz}$ ). 104.22 (s), 66.16 (s), 65.59 (s). $38.99(\mathrm{t}, J=22.07 \mathrm{~Hz}) .21 .86(\mathrm{~s}) . \mathrm{GC} \cdot \mathrm{MS}(\mathrm{ni} / \mathrm{e}$. relative intensity): $207(0.22) .150(0.77), 132(1.96), 127(0.11), 106$ (8.11), $105(100.00), 77(42.63) .57$ (23.50). 51 (23.20). IR $\left(\mathrm{CCl}_{4}\right): 3065.2928,2856,1720,1599.1452 .1375 \mathrm{~cm}^{-1}$.

5,5-Diethoxy-2,2-difluoro-4-iodo-1-phenyl-1-pentanone (tad). ${ }^{1} \mathrm{H}$ NMR $\left(\mathrm{CDCl}_{3}\right): \delta 8.10(\mathrm{~d} . J=7.79 \mathrm{~Hz}, 2 \mathrm{H}), 7.64$ (t. $J=7.79 \mathrm{~Hz}, \mathrm{lH}), 7.50(\mathrm{t}, J=7.79 \mathrm{~Hz} .2 \mathrm{H}), 4.31(\mathrm{~m} .2 \mathrm{H})$, 3.72 (m. 2H). 3.58 (m. 2H), 3.16 (m, lH). 2.87 (m. lH), 1.22 (q. $J=7.33,4 \mathrm{H}) .0 .86(\mathrm{~m}, 6 \mathrm{H}) .{ }^{19} \mathrm{~F} \mathrm{NMR}\left(\mathrm{CDCl}_{3}, \mathrm{CFCl}_{3}\right): \delta$ -107.53 (ddd, $J=278.64,17.08 .17 .08 \mathrm{~Hz}, \mathrm{IF}) \delta-104.27$ (ddd, $J=278.64,17.08,17.08 \mathrm{~Hz} . \mathrm{lF}){ }^{12} \mathrm{C} \mathrm{NMR}\left(\mathrm{CDCl}_{3}\right): \delta$ $188.52(\mathrm{t}, J=31.72 \mathrm{~Hz}) \cdot 134.47(\mathrm{~s}), 131.72(\mathrm{~s}), 130.28(\mathrm{t}$. $J=2.88 \mathrm{~Hz}), 128.78(\mathrm{~s}), 118.68(\mathrm{t} . J=256.72 \mathrm{~Hz}), 104.04$ (s). $64.19(\mathrm{~s}), 62.86(\mathrm{~s}), 38.82(\mathrm{t} . J=21.08 \mathrm{~Hz}) .30 .78(\mathrm{~s})$ $15.15(\mathrm{~s}), 15.13(\mathrm{~s})$. GC-MS (m/e, relative intensity): 373 (0.5). $309(0.02) .291(0.21), 271(0.12), 245(0.35) .225$ $(0.49), 180(0.73), 155(0.26), 149(0.56), 127(0.59), 104$ (56.10). $103(100.00) .83(38.56), 77(5.81) .75(52.17), 59$ (13. 59). 55 (64.39). IR $\left(\mathrm{CCl}_{4}\right): 3048,2988,2928,1712$. 1599. $1450.1376 \mathrm{~cm}^{-1}$.

Methyl 5,5-difluoro-3-iodo-6-0xo-6-phenylhexanoate (4ae). ${ }^{1} \mathrm{H}$ NMR $\left(\mathrm{CDCl}_{3}\right): \delta 8.10(\mathrm{~d}, J=7.79 \mathrm{~Hz}, 2 \mathrm{H}), 7.65$ (t. $J=7.10 \mathrm{~Hz}, 1 \mathrm{H}) .7 .51(\mathrm{t} . J=7.56 \mathrm{~Hz}, 2 \mathrm{H}), 4.48(\mathrm{~m} .2 \mathrm{H})$, $4.28(\mathrm{~m} .1 \mathrm{H}) .3 .01(\mathrm{~m} .2 \mathrm{H}), 2.06(\mathrm{~s}, 3 \mathrm{H}) .{ }^{19} \mathrm{~F} \mathrm{NMR}\left(\mathrm{CFCl}_{2}\right.$, $\mathrm{CDCl}_{3}$ ): $\delta-108.76$ (ddd. $\left.J=284.17 .14 .04 .14 .04 \mathrm{~Hz}, \mathrm{lF}\right) \delta$ -104.24 (ddd, $J=284.17$. 14.04. 14.04 Hz. IF). ${ }^{13} \mathrm{C}$ NMR $\left(\mathrm{CDCl}_{3}\right): \delta \mathrm{I} 88.27(\mathrm{t}, J=30.71 \mathrm{~Hz}), 170.22(\mathrm{~s}), 134.72(\mathrm{~s})$, $131.50(\mathrm{~s}), 130.33(\mathrm{t}, J=2.88 \mathrm{~Hz}) .128 .86(\mathrm{~s}), 118.61(\mathrm{t}$. $J=256.24 \mathrm{~Hz}), 68.91(\mathrm{~s}), 41.06(\mathrm{t} . J=24.04 \mathrm{~Hz}), 20.77(\mathrm{~s})$. 15.03 (t. $J=3.84 \mathrm{~Hz}$ ). IR $\left(\mathrm{CCl}_{4}\right): 3055.2924 .2854,1751$, 1703. $1450,1383 \mathrm{~cm}^{-1}$. GC-MS (m/e, relative intensity): $382\left(\mathrm{M}^{+} .0 .15\right), 35 \mathrm{l}(1.04), 323(2.85), 255(23.45) .105$ (100.00), $77(43.05)$

2,2-Difluoro-4-iodo-5-phenoxy-1-phenyl-1-hexanone (4af). ${ }^{1} \mathrm{H} \mathrm{NMR}\left(\mathrm{CDCl}_{3}\right): \delta 8.08(\mathrm{~d} . J=7.79 \mathrm{~Hz} 2 \mathrm{H}) .7 .64(\mathrm{t}$. $J=7.79 \mathrm{~Hz} \mathrm{lH}$ ). $7.49-6.98$ (m. 7H), 4.59 (quintet, $J=6.87$ $\mathrm{Hz} 1 \mathrm{H}$ ). 4.31 (dd $J=10.31 .5 .24 \mathrm{~Hz} 1 \mathrm{H}), 4.21$ (dd. $J=$ $10.31,6.87 \mathrm{~Hz} 1 \mathrm{H}$ ). 3.14 (dddd $J=16.27 \mathrm{~Hz}, 16.27 \mathrm{~Hz} .6 .87$ Hz. $6.87 \mathrm{~Hz} 2 \mathrm{H}$ ). ${ }^{19} \mathrm{~F} \mathrm{NMR}\left(\mathrm{CFCl}_{3}\right): \delta-106.77$ (ddd. $J=$ $283.15,16.27,16.17 \mathrm{~Hz}$. IF) $\delta-104.38$ (ddd. $J=283.15$, 16.27. 16.17 Hz. IF). ${ }^{13} \mathrm{C} \mathrm{NMR}\left(\mathrm{CDCl}_{3}\right): \delta 188.40$ (t. $J=$ 
$30.71 \mathrm{~Hz}), 157.79$ (s). 135.09 (s), $134.6 \mathrm{l}$ (s). 130.33 (t. $J=$ $2.88 \mathrm{~Hz}$ ). $129.64(\mathrm{~s}) .128 .82(\mathrm{~s}) .121 .67(\mathrm{~s}), 118.74$ (t. $J=$ $255.28 \mathrm{~Hz}$ ). 114.93 (s), 73.02 (s). 41.03 (t. $J=22.07 \mathrm{~Hz}$ ). 16.03 (s). GC-MS (n/e, relative intensity): 329 (11.54), 311 (0.56). $309(0.79) .291(1.51) .261(0.47) .247(1.65) .217$ (1.21). $183(3.21) .163(3.22) .139(5.69), 127(1.20), 119$ (8.16). $105(7.53), 93$ (42.16), 90 (12.28). 83 (50.52). 77 $(21.65), 65(32.46), 55(100.00)$. 51 (21.20). IR $\left(\mathrm{CCl}_{4}\right)$ : 3067. 3040, 2986, 2924, 2866, 1703. 1649. 1599. 1494. $1+54 \mathrm{~cm}^{-1}$.

2,2-Difluoro-t-iodo-1-phenyl-5-propanoxy-1-pentanone (4ag). ${ }^{1} \mathrm{H} \mathrm{NMR}\left(\mathrm{CDCl}_{3}\right): \delta 8.10(\mathrm{~d} . J=7.79 \mathrm{~Hz} .2 \mathrm{H}), 7.64$ (t. $J=7.33 \mathrm{~Hz} \mathrm{lH}$ ). 7.50 (t. $J=7.79 \mathrm{~Hz} 2 \mathrm{H}$ ). 4.41 (quintet. $J=6.42 \mathrm{~Hz} \mathrm{lH}), 3.71(\mathrm{dd}, J=10.54 .5 .50 \mathrm{~Hz}, 1 \mathrm{H}), 3.64$ (dd. $J=10.54 .6 .87 \mathrm{~Hz} .1 \mathrm{H}) .3 .42(\mathrm{~m}, 2 \mathrm{H}) .3 .17(\mathrm{~m}, \mathrm{lH})$. 2.87 (m. IH), 1.53 (sextet, $J=7.33 \mathrm{~Hz}, 2 \mathrm{H}$ ) 0.89 (t. $J=7.33$ Hz. $3 \mathrm{H}) .{ }^{19} \mathrm{~F} \mathrm{NMR} .\left(\mathrm{CDCl}_{3}, \mathrm{CFCl}_{3}\right): \delta-108.88$ (ddd. $J=$ $284.21,17.21$. 17.21 Hz, IF) $\delta-106.47$ (ddd. $J=284.21$. 17.21. 17.21 Hz. IF). ${ }^{13} \mathrm{C} \mathrm{NMR}\left(\mathrm{CDCl}_{3}\right): \delta 188.51$ (t. $J=$ $30.7 \mathrm{~Hz}$ ). 134.49 (s), 130.29 (t. $J=2.88 \mathrm{~Hz}), 128.78$ (s). $75.89(\mathrm{~s}) .72 .67(\mathrm{~s}) .40 .95(\mathrm{t}, J=22.55 \mathrm{~Hz}), 29.79(\mathrm{~s}) .22 .79$ $(\mathrm{s}) .18 .05(\mathrm{t} . J=2.88 \mathrm{~Hz}), 14.21(\mathrm{~s}), 10.64(\mathrm{~s}) . \mathrm{GC} \cdot \mathrm{MS}(\mathrm{m} / \mathrm{e}$. relative intensity): $323(0.44) .255(5.21), 213(10.28) .176$ (4.20). $156(0.84) .149(4.78) .147(3.64) .127(3.50) .105$ (100.00). $77(61.82), 57(8.68), 51$ (33.68). IR $\left(\mathrm{CCl}_{4}\right): 3055$. $2924.2854,1703,1599.1581 .1450 .1377 \mathrm{~cm}^{-1}$.

1-Cyclohexyl-2,2-difluoro-t-iodo-t-trimethylsilyl-1-butanone (4ba). ${ }^{1} \mathrm{H}$ NMR $\left(\mathrm{CDCl}_{3}\right): \delta 3.08$ (dd, $J=10.54 .3 .21$ Hz, 1H). 2.96 (dddd, $J=11.91,11.92,3.21 .3 .21 \mathrm{~Hz} .1 \mathrm{H}$ ). $2.60(\mathrm{~m}, 2 \mathrm{H}), 1.30-1.85(\mathrm{~m} . \mathrm{l0H}), 0.2 \mathrm{l}(\mathrm{s}, 9 \mathrm{H}) .{ }^{19} \mathrm{~F}$ NMR $\left(\mathrm{CDCl}_{3} \mathrm{CFCl}_{3}\right.$ ): $\delta-108.38$ (ddd, $J=279.45,16.55 .16 .55$ $\mathrm{Hz}, \mathrm{IF}) \delta-102.70$ (ddd. $J=279.45,16.55,16.55 \mathrm{~Hz}, \mathrm{IF}$ ). ${ }^{13} \mathrm{C} \mathrm{NMR}\left(\mathrm{CDCl}_{3}\right): \delta 203.90(\mathrm{t} . J=29.75 \mathrm{~Hz}), 118.20(\mathrm{t}$. $254.32 \mathrm{~Hz}$ ), 45.20 (s). 37.24 (t. $J=23.51 \mathrm{~Hz}$ ), 28.49 (s). 28.31 (s) $.25 .87(\mathrm{~s}), 25.70(\mathrm{~s}), 25.60(\mathrm{~s}), 5.03(\mathrm{t}, J=2.88$ $\mathrm{Hz}),-2.35$ (s). IR: $3393.2949,2926,1738.1451 \mathrm{~cm}^{-1}$. GCMS (m/e, relative intensity): $389(0.17), 388(0.89), 373$ (0.82). 261 (3.41). $177(0.12), 169$ (10.16). $127(2.20), 111$ (17.17). 83 (100.00). 73 (55.33). 55 (76.79). 53 (10.58).

Ethyl 5-cyclohexyl-4,4-difluoro-2-iodo-5-0xopentanoate (4bb). ${ }^{\circ} \mathrm{H}$ NMR $\left(\mathrm{CDCl}_{3}\right): \delta 4.52$ (dd. $J=10.54,3.67 \mathrm{~Hz}$. $\mathrm{lH}) .4 .21(\mathrm{q} . J=7.10 \mathrm{~Hz} .2 \mathrm{H}) .3 .10(\mathrm{~m} .1 \mathrm{H}) .2 .84(\mathrm{~m}, 1 \mathrm{H})$. $2.66(\mathrm{~m} .1 \mathrm{H}) .1 .90-1.25(\mathrm{~m} .10 \mathrm{H}) .1 .27(\mathrm{t} . J=7.33 \mathrm{~Hz} .3 \mathrm{H})$. ${ }^{13} \mathrm{C} \mathrm{NMR}\left(\mathrm{CDCl}_{3}\right): \delta 202.63$ (t. $\left.J=29.75 \mathrm{~Hz}\right), 170.80(\mathrm{~s})$. 117.04 (t. $J=255.28 \mathrm{~Hz}), 62.27(\mathrm{~s}) .44 .77(\mathrm{~s}) .40 .32(\mathrm{t}, J=$ $23.03 \mathrm{~Hz}$ ). 28.33 (s). 28.20 (s). 25.59 (s). 25.43 (s). 25.39 (s). 17.72 (s). 7.38 (t. $J=4.32 \mathrm{~Hz}) \cdot{ }^{19} \mathrm{~F} \mathrm{NMR}\left(\mathrm{CDCl}_{3}, \mathrm{CFCl}_{3}\right): \delta$ -107.18 (ddd. $278.44,21.32,11.29 \mathrm{~Hz}$. IF) $\delta-103.47$ (ddd. $278.44,21.32 .11 .29 \mathrm{~Hz}$, IF) IR $\left(\mathrm{CCl}_{4}\right): 2931,2857.1749$. 1601. 1484. $1283 \mathrm{~cm}^{-1}$. GC-MS (m/e. relative intensity): $388\left(\mathrm{M}^{+} .0 .25\right), 342(0.85) .323(1.45) .241(28.25) .111$ (100). $83(72.45)$.

1-Cyclohexyl-2,2-difluoro-4-(1.3-dioxolan-2-yl)-4-iodo1-butanone (4bc). ${ }^{~} \mathrm{H}$ NMR $\left(\mathrm{CDCl}_{3}\right): \delta 4.61$ (d. $J=3.21$ Hz, IH). $4.2 \mathrm{l}$ (ddd. $J=8.02 .5 .27 .3 .21 \mathrm{~Hz}, \mathrm{IH}$ ). 4.08 (n. $2 \mathrm{H}) .3 .95(\mathrm{~m}, 2 \mathrm{H}), 2.87(\mathrm{~m}, 2 \mathrm{H}), 2.69(\mathrm{~m}, 1 \mathrm{H}) .1 .78(\mathrm{~m}, 4 \mathrm{H})$. $1.29(\mathrm{~m} .6 \mathrm{H}) .{ }^{19} \mathrm{~F} \mathrm{NMR}\left(\mathrm{CDCl}_{j_{2}} \mathrm{CFCl}_{3}\right): \delta-107.6 \mathrm{l}$ (ddd. $J=$
$272.14,20.36 .10 .32 \mathrm{~Hz}$. IF) $\delta-103.22(\mathrm{ddd}, J=272.14$, 20.36. $10.32 \mathrm{~Hz} . \mathrm{IF}){ }^{13} \mathrm{C}$ NMR (CDCl $\mathrm{s}$. TMS): $\delta 203.00(\mathrm{t}$. $J=30.23 \mathrm{~Hz}), 117.58(\mathrm{t} . J=255.28 \mathrm{~Hz}), 104.14(\mathrm{~s}) .66 .11$ (s). $65.93(\mathrm{~s}), 44.86(\mathrm{~s}) .38 .0 \mathrm{l}(\mathrm{t}, J=23.03 \mathrm{~Hz}$ ). $28.37(\mathrm{~s})$, 28.33 (s), 25.65 (s). 25.49 (s). 25.42 (s), 22.08 (s). GC-MS $(\mathrm{m} / \mathrm{e}$, relative intensity): $261(0.74), 241(0.17), 168(0.14)$. $155(0.42) .127(0.57), 111(3.62), 106(1.20) .99(3.04), 83$ (21.51). $77(2.48), 74(3.40), 73(100.00) .55(30.53), 51$ (2.01). IR $\left(\mathrm{CCl}_{4}\right): 2978,2934.1735,1695.1598 .1450,1378$ $\mathrm{cm}^{-1}$.

1-Cyclohexyl-5,5-diethoxy-2,2-difluoro-4-iodo-1-pentanone (thd). ${ }^{1} \mathrm{H}$ NMR $\left(\mathrm{CDCl}_{3}\right): \delta 4.28$ (d. $J=3.67 \mathrm{~Hz}$. $\mathrm{lH}$ ). 3.70 (q. $J=7.33 \mathrm{~Hz}, 2 \mathrm{H}$ ). 3.57 (q. $J=7.33 \mathrm{~Hz} .2 \mathrm{H}$ ). 2.91 (m, $2 \mathrm{H}) .2 .67(\mathrm{~m} . \mathrm{lH}), 1.92-1.83(\mathrm{~m}, 6 \mathrm{H}) .{ }^{19} \mathrm{~F} \mathrm{NMR}\left(\mathrm{CDCl}_{3}\right.$. $\left.\mathrm{CFCl}_{3}\right): \delta$-107.21 (ddd. $J=272.24 .21 .32,10.35 \mathrm{~Hz}$, IF) $\delta$ -103.46 (ddd. $J=272.24,21.32,10.35 \mathrm{~Hz}, \mathrm{IF}$ ). ${ }^{13} \mathrm{C}$ NMR $\left(\mathrm{CDCl}_{3}\right.$ ): $\delta 203.29$ (t. $J=30.48 \mathrm{~Hz}$ ). 117.87 (t. $J=254.32$ Hz), 104.09 (s). $64.19(\mathrm{~s}), 63.91(\mathrm{~s}), 45.01$ (s). $37.48(\mathrm{t}, J=$ $23.03 \mathrm{~Hz}) .28 .44(\mathrm{~s}) .28 .31$ (s). $25.66(\mathrm{~s}) .25 .53(\mathrm{~s}) .25 .43(\mathrm{~s})$. $21.34(\mathrm{~s}), 15.17(\mathrm{~s}) . \mathrm{GC} \cdot \mathrm{MS}(\mathrm{m} / \mathrm{e}$, relative intensity): 373 $(0.56), 291(0.27), 225(0.46), 180(0.84), 177(0.33), 149$ $(0.60), 135(0.75), 127(0.62), 111(4.25) .103(100.00) .87$ $(8.55), 83(46.24), 77(4.46) .75(55.10) .67(5.68), 55$ (66.76). IR $\left(\mathrm{CCl}_{4}\right): 2976,2934,1734,1691$. 1599. 1450. 1375. $1275 \mathrm{~cm}^{-1}$.

Methyl-6-cyclohexyl-5,5-difluoro-3-iodo-6-oxohexanoate (the). ${ }^{1} \mathrm{H} \mathrm{NMR}\left(\mathrm{CDCl}_{3}\right): \delta 4.32$ (m. 2H). 2.91 (m. lH). 2.76 (m. 2H). 2.11 (s. 3H), 1.89-1.23 (m, 10H) ${ }^{19} \mathrm{~F} \mathrm{NMR} \mathrm{(CDCl}$. $\mathrm{CFCl}_{3}$ ): $\delta$-103.96 (ddd. $\left.J=285.25 .21 .94,13.97 \mathrm{~Hz}, \mathrm{IF}\right) \delta$ -107.40 (ddd. $J=285.25,21.94,13.97 \mathrm{~Hz}, \mathrm{IF}$ ). ${ }^{13} \mathrm{C}$ NMR $\left(\mathrm{CDCl}_{\mathrm{s}}\right): \delta 202.93(\mathrm{t}, J=29.75 \mathrm{~Hz}), 170.22(\mathrm{~s}) .117 .76(\mathrm{t}, J$ $=255.43 \mathrm{~Hz}$ ). 68.83 (s), 4.85 (s). 39.95 (t. $J=23.99 \mathrm{~Hz}$ ), $28.43(\mathrm{~s}), 28.33$ (s). $25.67(\mathrm{~s}), 25.49(\mathrm{~s}) .25 .42(\mathrm{~s}), 20.83(\mathrm{~s})$ 15.19 (t, $J=3.84 \mathrm{~Hz}$ ). GC-MS (m/e, relative intensity): 367 $(0.02), 261(2.05), 241(2.00), 201(0.51), 181(3.91), 161$ (1.34). $127(0.99) .111(25.34) .91(4.31), 83(100.00), 55$ (77.81). IR $\left(\mathrm{CCl}_{4}\right): 2934,2856.1747,1450.1381,1226 \mathrm{~cm}^{-1}$.

1-Cyclohexyl-2,2-difluoro-4-ioklo-5-phenoxy-1-pentanone (4hf). ${ }^{1} \mathrm{H}$ NMR $\left(\mathrm{CDCl}_{3}\right): \delta 7.28(\mathrm{~m}, 2 \mathrm{H}) .6 .99(\mathrm{t}, J=7.33$ Hz. $1 \mathrm{H}$ ). 6.90 (d. $J=7.79 \mathrm{~Hz}, 2 \mathrm{H}), 4.44(5, J=6.42 \mathrm{~Hz}$. $1 \mathrm{H}$ ). $4.24($ dd $J=10.31 .5 .27 \mathrm{~Hz}, 1 \mathrm{H}$ ). 4.15 (dd. $J=10.31$. $6.65 \mathrm{~Hz} .1 \mathrm{H}) .3 .03(\mathrm{~m} . \mathrm{lH}) .2 .92(\mathrm{~m} . \mathrm{lH}) .2 .78(\mathrm{~m} . \mathrm{lH}) .1 .86$ $-1.25(\mathrm{~m}, 10 \mathrm{H}) .{ }^{19} \mathrm{~F}$ NMR $\left(\mathrm{CDCl} \mathrm{C}_{2} \mathrm{CFCl}_{3}\right): \delta-106.78(\mathrm{ddd}, J$ $=270.28,19.40 .10 .35 \mathrm{~Hz}, \mathrm{IF}) \delta-104.30$ (ddd. $J=279.33$, $20.69,10.35 \mathrm{~Hz}, \mathrm{IF}) \cdot{ }^{13} \mathrm{C} \mathrm{NMR}\left(\mathrm{CDCl}_{3}\right): \delta 202.99$ (t. $J=$ $35.03 \mathrm{~Hz}), 157.86(\mathrm{~s}) .129 .69(\mathrm{~s}) .121 .73(\mathrm{~s}), 114.97(\mathrm{~s})$, 73.08 (s). 44.87 (s). 39.96 (t. $J=23.03 \mathrm{~Hz}$ ). 28.39 (s). 28.36 (s). 25.63 (s). 25.48 (s). 25.43 (s). 16.19 (s). GC-MS (m/e. relative intensity): $422\left(\mathrm{M}^{+}, 0.53\right), 330(2.59), 329(19.55)$, $291(2.88), 247(3.42), 217(3.05), 183(5.34), 163(4.95)$. $111(6.81) .94(34.74) .83(50.51), 77(23.80) .65(28.74) .55$ (100.00). 51 (20.65). IR $\left(\mathrm{CCl}_{4}\right): 3040.2932 .2856,1734$. 1559. $1496.1452 .1240 \mathrm{~cm}^{-1}$.

1-Cyclohexyl-2,2-difluoro-4-iodo-5-propanoxy-1-pentanone (4hg). ${ }^{1} \mathrm{H}$ NMR $\left(\mathrm{CDCl}_{3}\right): \delta 4.22$ (quintet. $J=6.65$ Hz. 1 H). $3.66(\mathrm{dd}, J=10.54,5.50 \mathrm{~Hz}, 1 \mathrm{H}), 3.58(\mathrm{dd}$, $J=10.54 .6 .87 \mathrm{~Hz} .1 \mathrm{H}) .3 .41$ (t. $J=6.87 \mathrm{~Hz}, 2 \mathrm{H}) .2 .87(\mathrm{~m}$. 
$2 \mathrm{H}), 2.62(\mathrm{~m} . \mathrm{HH}) .1 .56($ sextet, $J=7.33 \mathrm{~Hz} .2 \mathrm{H}) .1 .38-1.21$ (m, $10 \mathrm{H}), 0.90(\mathrm{t}, J=7.33 \mathrm{~Hz}, 3 \mathrm{H}) .{ }^{1.3} \mathrm{~F} \mathrm{NMR}\left(\mathrm{CDCl}_{3}\right.$. $\left.\mathrm{CFCl}_{3}\right): \delta-106.79$ (ddd. $\left.J=282.71 .19 .71,12.13 \mathrm{~Hz}, 1 \mathrm{~F}\right) \delta$ -104.23 (ddd, $J=282.71,19.73 .12 .14 \mathrm{~Hz} . \mathrm{IF}$ ). ${ }^{13} \mathrm{C}$ NMR $\left(\mathrm{CDCl}_{3}\right): \delta 203.06(\mathrm{t}, J=30.7 \mathrm{l} \mathrm{Hz}) .117 .71$ (t. $J=255.28$ Hz), $75.88(\mathrm{~s}), 72.70(\mathrm{~s}) .44 .86(\mathrm{~s}), 39.84(\mathrm{t}, J=20.03 \mathrm{~Hz})$. 28.38 (s). 28.30 (s), 25.63 (s), 25.47 (s). 25.40 (s), 22.80 (s). 10.60 (s). GC-MS (n/e. relative intensity): $329(0.09) .241$ (3.42). $181(3.48), 162(1.81), 133(2.28) .127(1.22), 111$ (18.09), 83 (97.38), $58(5.67) .55(100.00), 52(10.12)$. IR $\left(\mathrm{CCl}_{4}\right): 2934,2858,1734,1450.1289 \mathrm{~cm}^{-1}$.

Difluoro-1-iodo-1-trimethylsilyl-4-octanone (4ca). ${ }^{1} \mathrm{H}$ NMR $\left(\mathrm{CDCl}_{3}\right): \delta 3.08(\mathrm{dd}, J=10.54 \mathrm{~Hz}, 3.2 \mathrm{l} \mathrm{Hz}, \mathrm{IH}), 2.73$ (m, 2H). 2.59 (m, 2H), 1.61 (quintet, $J=7.33 \mathrm{~Hz}, 2 \mathrm{H}$ ). 1.34 (sextet, $J=7.33 \mathrm{~Hz} .2 \mathrm{H}$ ). 0.90 (t. $J=7.33 \mathrm{~Hz}, 3 \mathrm{H}$ ), 0.12 (s. $9 \mathrm{H}) .{ }^{19} \mathrm{~F} \mathrm{NMR}\left(\mathrm{CFCl}_{3}\right): \delta-109.15$ (ddd. $J=261.01 \mathrm{~Hz}$. 16.06. $16.06 \mathrm{~Hz}) \delta-103.79$ (ddd. $J=261.01 \mathrm{~Hz}, 16.06$. $16.06 \mathrm{~Hz}) \cdot{ }^{13} \mathrm{C} \mathrm{NMR}\left(\mathrm{CDCl}_{3}\right): \delta-2.40(\mathrm{~s}) .4 .89(\mathrm{~s}) .13 .82$ (s). 22.11 (s). 24.76 (s). 36.63 (s). 37.08 (t. $J=23.99 \mathrm{~Hz}$ ). 117.60 (t. $J=254.31 \mathrm{~Hz}), 201.15$ (t. $J=31.19 \mathrm{~Hz}$ ). IR $\left(\mathrm{CCl}_{4}\right): 2917,2904.1706,1448 \mathrm{~cm}^{-1}$. GC-MS (m/e. relative intensity): $55.00(54.27), 57.05$ (97.45), $73.05(100.00)$. $85.10(54.89), 101.05(6.33), 143.05(12.35) .151 .05(10.97)$. $157.10(8.31), 185.00(7.73) .235 .20(2.84), 362.15(0.75)$.

Ethyl +,4-difluoro-2-iodo-5-oxononanoate $(+\mathrm{cb}) .{ }^{1} \mathrm{H}$ NMR $\left(\mathrm{CDCl}_{3}\right): \delta 4.51(\mathrm{dd}, J=10.3 \mathrm{l} \mathrm{Hz} .3 .44 \mathrm{~Hz} .1 \mathrm{H}), 4.19$ (q. $J=7.33 \mathrm{~Hz}, 2 \mathrm{H}), 3.10(\mathrm{~m}, 2 \mathrm{H}), 2.63(\mathrm{t} . J=6.42 \mathrm{~Hz} .3 \mathrm{H})$. 1.56 (quintet, $J=7.33 \mathrm{~Hz} .2 \mathrm{H}$ ). 1.31 (sextet. $J=7.33 \mathrm{~Hz}$. $2 \mathrm{H}) .1 .25$ (t. $J=7.33 \mathrm{~Hz} .2 \mathrm{H}) .0 .89$ (t. $J=7.33 \mathrm{~Hz}, 3 \mathrm{H}){ }^{19} \mathrm{~F}$ NMR $\left(\mathrm{CFCl}_{3}\right): \delta-108.37$ (ddd. $J=272.14,17.37,17.37$ Hz, IF). $\delta-103.54$ (ddd, $J=272.14 .17 .37 .17 .37 \mathrm{~Hz}, 1 \mathrm{~F}$ ). ${ }^{13} \mathrm{C}$ NMR $\left(\mathrm{CDCl}_{3}\right): \delta 199.94(\mathrm{t} . J=30.71 \mathrm{~Hz}) .170 .77(\mathrm{~s})$. $116.48(\mathrm{t} . J=255.28 \mathrm{~Hz}), 62.57(\mathrm{~s}), 39.96(\mathrm{t} . J=23.03 \mathrm{~Hz})$. 35.77 (s), 24.64 (s), 22.08 (s). 13.75 (s). 7.69 (t. $J=3.84$ $\mathrm{Hz})$. GC-MS (m/e. relative intensity): $362\left(\mathrm{M}^{+} .0 .03\right) .316$ $(0.43) .269(0.41) .235(0.40) .215(5.53) .141(1.61) .127$ $(2.47) .122(2.43), 101(1.24), 93(1.83), 85$ (69.22). 77 (4.20). $57(100.00) .55(24.92) .51$ (9.13). IR $\left(\mathrm{CCl}_{4}\right): 2976$. 2935. 1734.1690.1597.1450 $\mathrm{cm}^{-1}$.

3,3-Difluoro-1-(1,3-dioxolan-2-yl)-1-iodo-4-octanone (4cc). ${ }^{1} \mathrm{H} \mathrm{NMR}\left(\mathrm{CDCl}_{3}\right): \delta 4.65(\mathrm{~d} . J=3.21 \mathrm{~Hz}, \mathrm{lH}) .4 .22$ (m. 1H). $4.10(\mathrm{~m} .2 \mathrm{H}) .3 .96(\mathrm{~m} .2 \mathrm{H}) .2 .88(\mathrm{~m} . \mathrm{lH}) .2 .73(\mathrm{~m}$. lH). 2.70 (t. $J=2.87 \mathrm{~Hz}, 2 \mathrm{H}$ ). 1.61 (quintet. $J=7.33 \mathrm{~Hz}$. 2H). 1.34 (sextet. $J=7.33 \mathrm{~Hz}, 2 \mathrm{H}$ ) 0.92 (t. $J=7.33 \mathrm{~Hz}$. $3 \mathrm{H}){ }^{119} \mathrm{~F} \mathrm{NMR}\left(\mathrm{CFCl}_{3}\right): \delta-106.32$ (ddd. $J=282.04,15.78$. $15.78 \mathrm{~Hz}$. IF) $\delta-104.80$ (ddd. $J=286.25,18.75 .17 .89 \mathrm{~Hz}$. lF). ${ }^{13} \mathrm{C}$ NMR $\left(\mathrm{CDCl}_{3}\right): \delta 200.34(\mathrm{t}, J=30.7 \mathrm{l} \mathrm{Hz}), 117.02$ $(254.32 \mathrm{~Hz}) .104 .18(\mathrm{~s}) .66 .13(\mathrm{~s}) .65 .91$ (s). 37.70 (t. $J=$ $23.51 \mathrm{~Hz}$ ). 36.08 (s). 29.78 (s). 22.13 (s). 21.77 (t. $J=2.88$ Hz). 13.08 (s). GC-MS (m/e. relative intensity): $362\left(\mathrm{M}^{+}\right.$. $0.10), 235(0.02), 127(0.52), 119(0.26), 99(3.37), 85$ $(8.75) .77(2.34) .74(4.27), 73(100.00), 57$ (20.96). 55 (9.64). 51 (2.44). IR $\left(\mathrm{CCl}_{4}\right): 2959.2928 .1741 .1464 .1379$. $1259 \mathrm{~cm}^{-1}$.

1,1-Diethoxy-4,+-difluoro-2-iodo-5-nonanone (4cd). ${ }^{1} \mathrm{H}$ NMR $\left(\mathrm{CDCl}_{3}\right): \delta 4.30$ (d. $J=4.12 \mathrm{~Hz} .1 \mathrm{H}$ ). 4.15 (quintet. $J$ $=4.35 \mathrm{~Hz} . \mathrm{lH}) .3 .70(\mathrm{~m}, 2 \mathrm{H}) .3 .57(\mathrm{~m} .2 \mathrm{H}), 2.94(\mathrm{~m}, \mathrm{lH})$.
2.69 (t. $J=7.33 \mathrm{~Hz} .3 \mathrm{H}), 1.35$ (sextet. $J=7.33 \mathrm{~Hz}, 2 \mathrm{H}$ ), $1.25-1.21$ (m. 8H). 0.92 (t. $J=7.33 \mathrm{~Hz} .3 \mathrm{H}$ ). ${ }^{19} \mathrm{~F}$ NMR $\left(\mathrm{CDCl}_{3}, \mathrm{CFCl}_{3}\right): \delta-107.33(\mathrm{ddd}, J=277.04 .20 .89 .13 .63$ Hz. IF) $\delta-105.10($ ddd, $J=277.32,20.88 .13 .64 \mathrm{~Hz}$, IF). ${ }^{13} \mathrm{C}$ NMR $\left(\mathrm{CDCl}_{3}\right): \delta 200.54(\mathrm{t} . J=32.63 \mathrm{~Hz}), 117.33(\mathrm{t} . J=$ $253.36 \mathrm{~Hz}$ ). $104.12(\mathrm{~s}) .64 .26(\mathrm{~s}) .63 .88(\mathrm{~s}) .37 .27(\mathrm{t}, J=$ $23.99 \mathrm{~Hz}), 36.26(\mathrm{~s}), 29.78(\mathrm{~s}), 22.15(\mathrm{~s}), 21.17(\mathrm{t} . J=3.84$ $\mathrm{Hz}), 15.17(\mathrm{~s}), 15.13(\mathrm{~s}), 13.84(\mathrm{~s})$. GC-MS (m/e. relative intensity): 347 (1.009), $219(0.43), 191$ (0.51), 172 (1.95), 169 (1.35), 134 (1.58). $127(0.89) .115(2.20) .105(5.59)$. 103 (100.00), $87(11.43) .85(31.59) .77(7.80), 75(84.06)$. $57(76.10), 55(24.31), 51(6.73)$. IR $\left(\mathrm{CCl}_{4}\right): 2962.2934$. 1736. $1412.126 \mathrm{~cm}^{-1}$.

Methyl 5,5-difluoro-3-iodo-6-oxodecanoate (4ce). ${ }^{1} \mathrm{H}$ NMR $\left(\mathrm{CDCl}_{3}\right): 4.35(\mathrm{~m} .2 \mathrm{H}), 4.29(\mathrm{dd} . J=10.31,6.42 \mathrm{~Hz}$, 1H). 4.24 (dd. $J=10.31 .5 .04 \mathrm{~Hz}, 1 \mathrm{H}), 2.84-2.74(\mathrm{~m}, 2 \mathrm{H})$, 2.70 (t. $J=7.33 \mathrm{~Hz}, 2 \mathrm{H}), 2.11$ (s, 3H), 1.61 (quintet, $J=$ $7.33 \mathrm{~Hz}, 2 \mathrm{H}$ ), 1.35 (sextet, $J=7.33 \mathrm{~Hz}, 2 \mathrm{H}$ ). 0.92 (t. $J=$ $7.33 \mathrm{~Hz} .3 \mathrm{H}$ ). ${ }^{19} \mathrm{~F} \mathrm{NMR}\left(\mathrm{CDCl}_{3} . \mathrm{CFCl}_{3}\right): \delta-107.72$ (ddd. $J$ $=286.58,20.47,13.03 \mathrm{~Hz}$. IF) $\delta-104.68$ (ddd. $J=286.49$, $20.44,13.06 \mathrm{~Hz}, \mathrm{lF}),{ }^{13} \mathrm{C}$ NMR $\left(\mathrm{CDCl}_{3}\right): \delta 200.23(\mathrm{t}, J=$ $31.15 \mathrm{~Hz}), 170.22(\mathrm{~s}), 116.85(\mathrm{t}, J=255.28 \mathrm{~Hz}) .68 .83(\mathrm{~s})$, 39.67 (t. $J=23.03 \mathrm{~Hz}$ ). 35.93 (s). 24.73 (s). 22.12 (s). 20.82 (s). 15.01 (t. $J=4.32 \mathrm{~Hz}), 13.81$ (s). GC-MS (m/e. relative intensity): 235 (4.38). $155(2.96), 127(0.58), 113(0.72), 90$ (4.43). 85 (67.84). 77 (1.67). $71(2.18) .59(5.66) .57(100.00)$, 51 (6.94). IR $\left(\mathrm{CCl}_{4}\right): 2961.2934,1745,1461381,1226$, $1037 \mathrm{~cm}^{-1}$.

4,t-Difluoro-2-iodo-1-phenoxy-5-nonanone $(+\mathrm{cf}) .{ }^{1} \mathrm{H}$ NMR $\left(\mathrm{CDCl}_{3}\right): \delta 7.29(\mathrm{~m}, 2 \mathrm{H}) .6 .99(\mathrm{t} . J=7.33 \mathrm{~Hz} . \mathrm{lH}), 6.89$ (d. $J=7.79 \mathrm{~Hz} .2 \mathrm{H}), 4.43($ dt. $J=11.91 .6 .07 \mathrm{~Hz}, 1 \mathrm{H}$ ), 4.25 $($ dd. $J=10.08 .5 .04 .1 \mathrm{H}) .4 .15(\mathrm{dd}, J=10.08 .6 .87 .1 \mathrm{H}) .3 .03$ $(\mathrm{m}, \mathrm{lH}), 2.78(\mathrm{~m}, \mathrm{lH}), 2.7 \mathrm{l}(\mathrm{t} . J=7.33 \mathrm{~Hz}, 2 \mathrm{H}), 1.58$ (quintet, $J=7.33 \mathrm{~Hz} .2 \mathrm{H}$ ), 1.33 (sextet, $J=7.33 \mathrm{~Hz}, 2 \mathrm{H}) .0 .9 \mathrm{l}$ (t. $J=$ $7.33 \mathrm{~Hz} .3 \mathrm{H}) .{ }^{19} \mathrm{~F} \mathrm{NMR}\left(\mathrm{CDCl}_{3} \mathrm{CFCl}_{3}\right): \delta-106.79$ (ddd. $J=$ $283.25,17.95 .17 .96 \mathrm{~Hz}$. IF) $\delta-105.22(\mathrm{ddd}, J=283.25$, $17.95,17.96 \mathrm{~Hz}, \mathrm{lF}) .{ }^{13} \mathrm{C}$ NMR $\left(\mathrm{CDCl}_{3}\right): \delta 200.25(\mathrm{t}, J=$ $32.15 \mathrm{~Hz}$ ). $129.70(\mathrm{~s}) .121 .76(\mathrm{~s}) .117 .04(\mathrm{t} J=255.28 \mathrm{~Hz}$ ). 114.97 (s). 73.07 (s). 39.72 (t. $J=23.03 \mathrm{~Hz}$ ). 35.98 (s). 24.69 (s). 22.12 (s). 16.00 (t. $J=4.32$ ). 13.81 (s). GC-MS (n/e, relative intensity): $303(7.58) .177$ (1.17), $156(28.22)$. $133(2.04) .105(2.96) .94(61.80) .85(46.08) .77(21.03) .64$ (34.49). $57(100.00) .51$ (26.26). IR $\left(\mathrm{CCl}_{4}\right): 3040.2961$, 2932. 1741. 1599. 1496, $1464,1383 \mathrm{~cm}^{-1}$.

t,t-Difluoro-2-iodo-1-propanoxy-5-nonanone $(4 \mathrm{cg}) .{ }^{~} \mathrm{H}$ $\operatorname{NMR}\left(\mathrm{CDCl}_{3}\right): \delta 4.25(\mathrm{~m} . \mathrm{lH}) .3 .67$ (dd. $J=10.54 .5 .50 \mathrm{~Hz}$, 1H). 3.58 (dd. $J=10.54 .7 .33 \mathrm{~Hz} . \mathrm{lH}) .3 .42(\mathrm{~m}, 2 \mathrm{H}), 2.93$ (m. $1 \mathrm{H}), 2.69(\mathrm{t} . J=7.33 \mathrm{~Hz}, 2 \mathrm{H}), 2.66(\mathrm{~m}, 1 \mathrm{H}), 1.59(\mathrm{~m}, 4 \mathrm{H})$. 1.35 (sextet. $J=7.33 \mathrm{~Hz}, 2 \mathrm{H}$ ). 0.92 (t. $J=7.33 \mathrm{~Hz} .6 \mathrm{H}$ ). ${ }^{19} \mathrm{~F}$ NMR $\left(\mathrm{CDCl}_{2}, \mathrm{CFCl}_{3}\right): \delta-106.63$ (ddd. $J=281.04$. 15.37, $15.37 \mathrm{~Hz}$. IF) $\delta-105.28$ (ddd, $J=281.04,15.37 .15 .37 \mathrm{~Hz}$, IF) ${ }^{13} \mathrm{C}$ NMR $\left(\mathrm{CDCl}_{3}\right): \delta 200.39(\mathrm{t}, J=30.71 \mathrm{~Hz}) .117 .16$ (t. $J=25.43 \mathrm{~Hz}$ ). $75.88(\mathrm{~s}) .72 .72$ (s). 39.68 (t. $J=23.03$ Hz). 36.07 (s). 24.73 (s). 22.82 (s). 22.15 (s). 18.04 (t. $J=$ $4.80 \mathrm{~Hz}$ ). 13.84 (s). 10.63 (s). GC-MS (n/e. relative intensity): 303 (0.17). 235 (1.55). $193(0.69) .177$ (0.83). 173 $(4.05), 156(1.87), 127(1.75), 111(4.03) .93(5.15), 85$ 
(72.28). 77 (2.19), $57(100.00) .51$ (8.59). IR ( $\left.\mathrm{CCl}_{4}\right): 2934$. 2957. $1734,1453,1425,1361 \mathrm{~cm}^{-1}$.

Acknowledgment. This work was supported by Wonkwang University in 2003.

\section{References}

1. Filler, R: Kobavashi. Y. Biochemical Aspects of Fltorine Chemishy: Elsevier Biochemical Press and Kodansha Ltd.: 1982: b) Filler. R. Biochentistry Inolving Carbon-Flworine Bonds. ACS: Washington D. C. 1976

2. Welch. J. J. Tetrohedron 1987, 43.3123.

3. Gelb, M. H.: Svaren. I. P: Abeles. R. H. Biochem. 1985, 24. 1814

4. Burkholder C. R.: Dolbier, W. R: Medebielle. M. J. F/torine Chem. 2000. 102.369.

5. Davis. C. R.: Burton. D. J.: Yang. Z. Y. J. Flonine Chem. 1995. 70. 135 .

6. Elsheimer, S.: Dolbier. W. R: Muria. M. J. Org. Chem. 1984, 49. $20 j$

7. (a) Rong, G: Keese. R. Tetrahedron Lett. 1990. 31, 5615. (b)
Huang. W. Y.: Wang. W.: Hung. B. N. Acta Chimical Sinta (Engl. $E d)$.

8. Hung. W. Y.: Zhan1g. H. J. J. Fluorine Chent. 1990. 50. 133.

9. Lang. R. W: Schaud, B. Tetrahedron Lett. 1988. 29. 2943.

10. Watanabe. S.; Fajita. T.: Sakamoto. M: Takeda, H.: Kitazume, T:; Yamazaki. T. J. Fluorime Chem, 1997,82, 1.

11. (a) Qiu. Z.: Button. D. I. J. Org. Chent 1995.60.3465. (b) Yang. Z. Y.: Buton. D. I. J. Org. Chem. 1991. 56. 170. (c) Yang. Z. Y.: Burtont. D. T. J. Flnotine Chent 1989. 45.435.

12. Chen. J.: Hu, C. M. J. Chem. Soc. Perkin Trans 1 1994, 1111.

13. Qiu. Z. M.: Burton. D. J. Tetrahedron Lett. 1994, 35. 1813.

14. (a) Yang. Z. Y.; Burton, D. J. J. Org. Chem. 1992. 57. 5144. (b) Qiu. Z. M.: Button. D. T. Tetrohedhon Lett. 1993. 34. 3239.

15. Kwak. K. C.: Oh. H. C.: Yun. Y. G.: Kim. B. H.: Lee. Y. H.: Chai. K. Y. Bull. Korream Chem. Soc. 2002. 23(1). 157.

16. (a) Kuroboshi, M.: Ishihara. T. Tetahedron Lett. 1987, $28,6481$. (b) Kuroboshi. M:- Ishihara, T. Bull. Chem. Soc. Jpn. 1990. 63 428 .

17. Chen1. Q. Y.: Yang. Z. Y.: Zhao. C. X.: Qiu. Z. M. J. Chent. Soc. Perkin Trans l 1998. 563

18. (a) Welch. J. T. Selectne Fluorination in Organic and Bioorganic Chemistry, ACS: Washington. DC, 1991. (b) Yang. Z. Y; Burton. D. J. Tetohedron Lett. 1991, 32, 1019. (c) Kitagawa. O; Mura, A.; Kobayashi, Y; Taguchi. T. Chem Lett. 1990, 1011. 